\title{
Oscillatory thermopower of carbon chains: First-principles calculations
}

\author{
Bin Wang, ${ }^{1}$ Yanxia Xing, ${ }^{1}$ Langhui Wan, ${ }^{2}$ Yadong Wei, ${ }^{2}$ and Jian Wang ${ }^{3,1, *}$ \\ ${ }^{1}$ Key Laboratory of Materials Physics, Institute of Solid State Physics, Chinese Academy of Sciences, \\ Hefei, Anhui, People's Republic of China \\ ${ }^{2}$ Department of Physics, School of Science, Shenzhen University, Shenzhen, People's Republic of China \\ ${ }^{3}$ Department of Physics, The University of Hong Kong, Pokfulam Road, Hong Kong
}

(Received 29 November 2004; published 30 June 2005)

\begin{abstract}
We investigate the thermoelectric transport through carbon chains connected by two Al leads. Using a Landauer-Buttiker-like formula, we calculate the thermopower and thermoconductance of $\mathrm{Al}-\mathrm{C}_{n}-\mathrm{Al}$ from first principles. We find that the charge transfer plays an important role in the thermoelectric transport. Because of the charge transfer, the thermopower changes sign for even-odd number of carbon atoms. The thermopower and electric conductance as a function of the gate voltage also exhibit oscillatory behaviors with a phase difference of $\pi / 2$.
\end{abstract}

DOI: 10.1103/PhysRevB.71.233406

PACS number(s): 73.40.Jn, 72.15.Jf, 73.40.Cg, 71.15.Mb

The quantum transport through atomic junctions has attracted increasing attention recently. ${ }^{1}$ To capture the atomic degrees of freedom, one has to use the first-principles calculation in the theoretical modeling of atomic systems. ${ }^{2-10} \mathrm{Up}$ to now, most of the theoretical studies on transport properties through atomic systems focus on the conductance and the $I-V$ characteristics. Less attention has been paid on the thermoelectric transport. The thermoelectric properties of an atomic system is important since it provides more information on the transport properties. For instance, at low temperatures, the electric conductance is proportional to the transmission coefficient at Fermi level while the thermopower is related to the derivative of the transmission coefficient so that the sign of the thermopower determines the sign of effective charge carriers. ${ }^{11}$ In this work, we investigate the thermoelectric properties of atomic junctions from first principles. In particular, we calculate the thermopower and thermoconductance of a series of carbon chains with different lengths connected by $\mathrm{Al}$ leads. We find that the charge transfer ${ }^{4}$ plays an important role in the thermoelectric transport. Due to the charge transfer during the formation of the $\mathrm{Al}-\mathrm{C}_{n}-\mathrm{Al}$ junction, the thermopower changes sign for evenodd number of carbon atoms. Since the charge transfer can be critically controlled by the gate voltage, we investigate the thermopower and the electric conductance as a function of the gate voltage which also show oscillatory behaviors with a phase difference being approximately $\pi / 2$. Roughly speaking, for even-number carbon chains, the electric conductance reaches maximum when the charge transfer is 2 and is minimum when there is no charge transfer. When the charge transfer is 1 the thermopower is maximum whereas the transferring of three charges leads to a minimum of the thermopower. All these behaviors can be described within the picture of $\pi$ orbitals conduction of carbon chains.

For the carbon chains with Al leads, the quantum transport problem is solved using the ab initio technique of Ref. 5 where DFT is carried out within the nonequilibrium Green's function (NEGF) formalism. We use an $s, p, d$ real-space linear combination of atomic orbitals (LCAO) basis set ${ }^{5,7}$ and define the atomic core by standard nonlocal norm conserving pseudopotential. ${ }^{12}$ The density matrix of the device is constructed via NEGF and the semi-infinite leads provide real-space potential boundary conditions for the Kohn-Sham (KS) potential of the device scattering region: this region consists of the molecule plus several layers of the metal leads. The KS potential includes contributions from Hartree, exchange, correlation, the atomic core, and any other external potentials. The NEGF-DFT iteration is numerically converged to $10^{-4} \mathrm{eV}$ which we determine to be reasonable for our purpose. The NEGF-DFT formalism has several good features for our purpose: ${ }^{5,7}$ (i) it constructs charge density under external bias potential using NEGF, thereby treats open device structures within the full self-consistent atomistic model of DFT; (ii) it treats atoms in the device and the leads at equal footing; (iii) it treats localized and scattering states at equal footing; (iv) it is numerically efficient. The NEGF-DFT formalism ${ }^{5,7}$ is now a standard tool for $a b$ initio atomistic calculations of transport and we refer interested readers to the existing literature. ${ }^{5,7}$

To analyze the thermoelectric effect, we apply a voltage bias $V_{b}$ and a temperature gradient $\Delta T$ between two atomic leads such that there is no electric current $I_{e}$ through the system. The thermopower is defined as the ratio between the voltage bias and the temperature gradient. With this definition, one finds ${ }^{13}$

$$
S(\mu)=-\frac{1}{e T} \frac{K_{1}(\mu)}{K_{0}(\mu)},
$$

where $e$ is positive and

$$
K_{n}=\int d E\left[-\partial_{E} f(E)\right](E-\mu)^{n} \mathcal{T}(E)
$$

with $\mathcal{T}(E)$ the transmission coefficient. Here the left lead is maintained at temperature $T$ and bias $V$ and the right lead is at $T+\Delta T$ and $V+V_{b}$. Although the electric current vanishes, there is a heat current $I_{q}$ which is proportional the temperature gradient, i.e., $I_{q}=\kappa \Delta T$, where $\kappa$ is the thermoconductance given by ${ }^{14}$ 


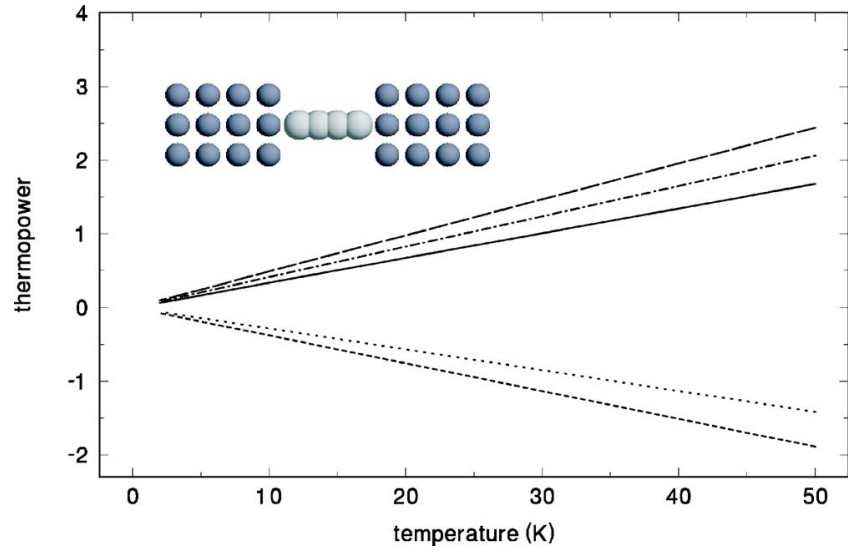

FIG. 1. (Color online) The thermopower vs temperatures for Al- $\mathrm{C}_{n}$-Al junction with $n=5$ (solid line), $n=6$ (dotted line), $n=7$ (dot-dashed line), $n=8$ (short dashed line), and $n=9$ (long dashed line). The unit of the thermopower is $\mu \mathrm{V} / \mathrm{K}$ and the temperature is measured in K. Right inset: schematic plot of the $\mathrm{Al}-\mathrm{C}_{5}-\mathrm{Al}$ device.

$$
\kappa(\mu)=\frac{2}{h T}\left(K_{2}(\mu)-\frac{K_{1}^{2}(\mu)}{K_{0}(\mu)}\right) .
$$

In the low temperatures, one can expand Eqs. (1) and (3) to get $^{14}$

$$
S(\mu)=-\frac{\pi^{2} k_{B}^{2} T}{2 e}\left[\frac{\partial \ln G(\mu)}{\partial \mu}+\frac{\pi^{2} k_{B}^{2} T^{2}}{15 G(\mu)} \frac{\partial^{3} G(\mu)}{\partial \mu^{3}}\right]+\cdots
$$

and

$$
\begin{aligned}
\kappa(\mu)= & \frac{\pi^{2} k_{B}^{2} T}{2 e^{2}} G(\mu)\left\{1+\frac{\pi^{2} k_{B}^{2} T^{2}}{15}\left[\frac{8}{G(\mu)} \frac{\partial^{2} G(\mu)}{\partial \mu^{2}}\right.\right. \\
& \left.\left.-5\left(\frac{\partial G(\mu)}{\partial \mu}\right)^{2}\right]\right\}+\cdots .
\end{aligned}
$$

Therefore, at the low temperatures, the sign of the thermopower is determined by the derivative of the electric conductance $G(\mu)$ while the thermoconductance is proportional to the electric conductance which is the Wiedemann-Franz law. In the numerical calculation, the energy is measured in a.u. $(1 \mathrm{a} \cdot \mathrm{u} .=27.2 \mathrm{eV})$.

Now we consider carbon chains with $n$ carbon atoms connected by two $\mathrm{Al}$ atomic leads ${ }^{15}$ (see Fig. 1).

In Fig. 1 we plot the thermopower as a function of the temperature $T$ for different $n$. We see that the thermopower is largely linear in $T$. For odd-number carbon chains, the thermopower is positive while for even-number carbon chains, it reverses its sign. We attribute this behavior to filling of the $\pi$ state. It is known that the transport through carbon chains is dominated by the $\pi$ state which is double degenerate. For $\mathrm{Al}-\mathrm{C}_{n}-\mathrm{Al}$ carbon chains, each carbon atom contributed two electrons to the $\pi$ states. $^{3}$ As a result, for $\mathrm{Al}-\mathrm{C}_{n}-\mathrm{Al}$ carbon chains with odd $n$, the highest occupied molecular orbital (HOMO) is half filled leading to a large conductance while for even $n$ the HOMO is completely filled making the conductance very small. ${ }^{18}$ In addition, due to the electronegative nature of the carbon atoms the charge is transferred from the leads to the carbon atoms. ${ }^{4}$ Since the charge transfer is criti-

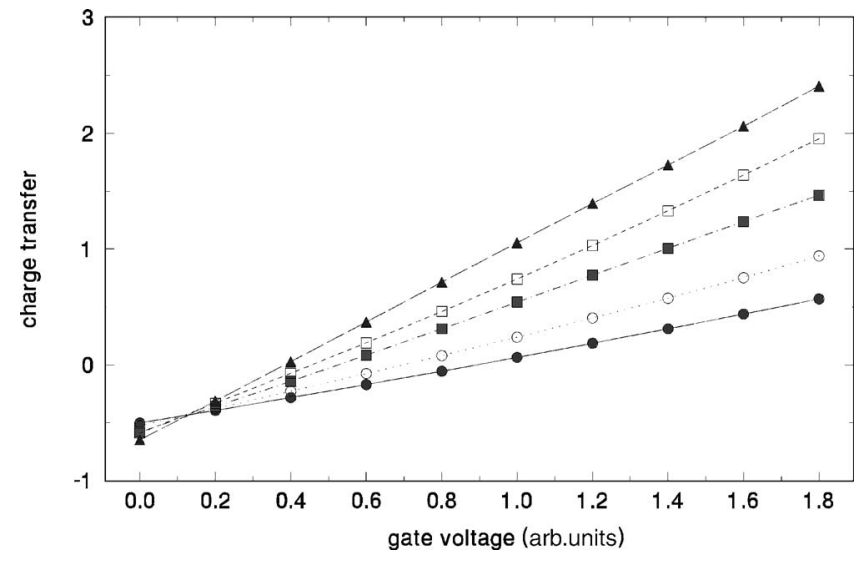

FIG. 2. The charge transfer vs gate voltage (in a.u.) for different carbon chains with $n=5$ (solid circle), $n=6$ (open circle), $n=7$ (solid square), $n=8$ (open square), and $n=9$ (triangle).

cally controlled by the gate voltage, we plot in Fig. 2 the charge transfer as a function of the gate voltage for different carbon chains. As expected, the charge transfer is linearly proportional to the gate voltage. In the absence of the gate voltage, about $-0.5 e$ (half an electron) has been transferred from the leads to the carbon atoms (see Fig. 2). Hence for odd-number chains, the HOMO is more than half filled so that charge transfer makes the conductance smaller. On the other hand, for even-number chains, there is a $\pi$ state which is partially occupied by about $-0.5 e$ (one-eighth of the filling since the HOMO can accommodate four electrons) so that the conductance increases due to the charge transfer. This can be seen clearly from Fig. 3 where we plot the electric conductance of different carbon chains as a function of the gate voltage. From the above argument, we see that the derivative of the conductance with respect to the chemical potential is negative for odd-number chains and positive for even-number chains. For our atomic system the energy scale is of order of $\mathrm{eV}$ so that for the temperature of order $100 \mathrm{~K}$ is still in the low-temperature limit. Hence from Eq. (4), we conclude that the thermopower is positive (negative) for odd (even) number of carbon atoms in the absence of gate voltage. This physical picture agrees qualitatively with our nu-

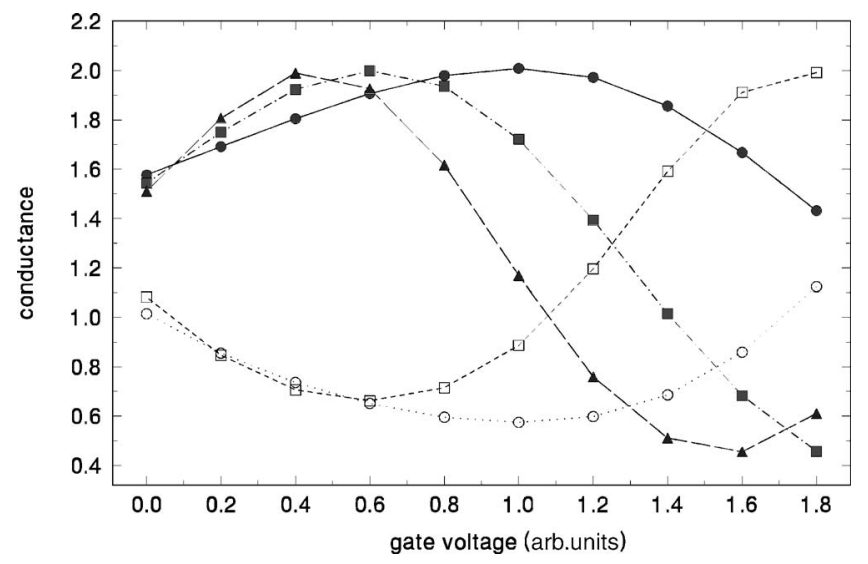

FIG. 3. The electric conductance vs gate voltage for different carbon chains. Here the symbols are the same as in Fig. 2. 


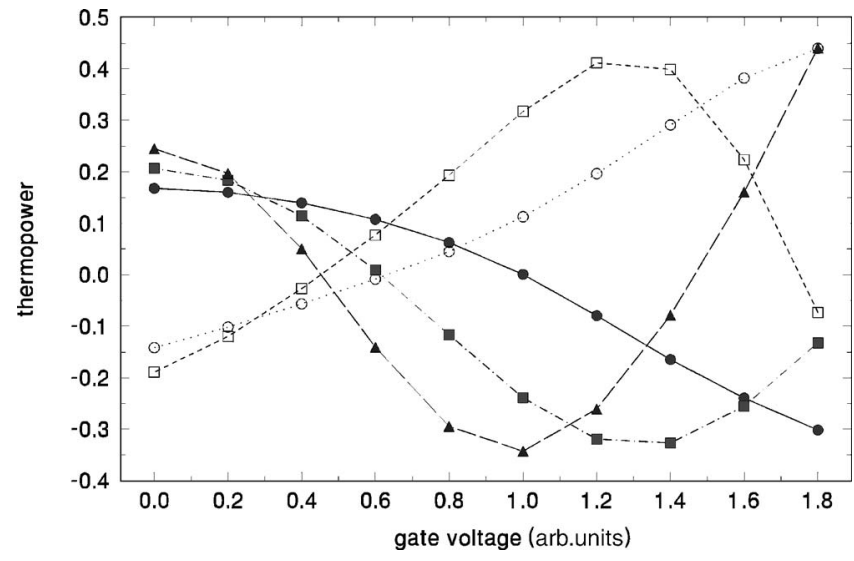

FIG. 4. The thermopower at $5 \mathrm{~K}$ vs gate voltage for different carbon chains. Here the symbols are the same as in Fig. 2.

merical calculation depicted in Fig. 4 where we plot the thermopower vs the gate voltage. ${ }^{21}$ When the positive gate voltage is applied, electrons are depleted from the region where carbon atoms are located. As the gate voltage is increased such that the $-0.5 e$ charge, which has been transferred into the region of carbon atoms due to the formation of the $\mathrm{Al}-\mathrm{C}_{n}-\mathrm{Al}$ chain, is completed depleted. At this gate voltage $v_{0},{ }^{22}$ the conductance should reach maximum for odd-number chains due to the half filled $\pi$ state while for even-number chains the conductance goes to minimum because of the completely filled $\pi$ states. This is indeed what we observed in Fig. 3. Furthermore, the thermopower is zero at $v_{0}$. From Figs. 3 and 4 , we see that the zeros of thermopower are well correlated with the zeros of charge transfer. Upon further increasing the gate voltage, the carbon atoms are positively charged indicating the electrons have been transferred from the carbon atoms to the leads. In this case, for even-number chains, this charge transfer from the filled $\pi$ states gives rise to "hole" state resulting in an increase of the electric conductance or positive thermopower. For oddnumber chains, the electron occupation is less than the half filling and the conductance decreases making thermopower positive. From Fig. 3, we also see that the even-odd parity oscillation for the conductance persists when the gate voltage is less than 0.8 a.u. beyond which this oscillation breaks down. We also note that the even-odd parity oscillation for the electric conductance and the thermopower may not occur simultaneously. They are determined by the charge transfer. However, the $\pi / 2$ phase shift between the oscillation of electric conductance and thermopower remains. To see this more clearly, we plot in Fig. 5 the charge transfer, conductance, and thermopower as a function of the gate voltage for an $\mathrm{Al}-\mathrm{C}_{10}-\mathrm{Al}$ chain. We see a clear oscillation of electric conductance and thermopower as the gate voltage is varied. In particular, when $v_{g} \approx 0.4 \mathrm{a}$.u. the charge transfer is zero so that the $\pi$ states are completely filled for $\mathrm{C}_{10}$. At this gate voltage the electric conductance is minimum and the corresponding thermopower is zero. When $v_{g}<0.4 \mathrm{a}$.u. the system shows holelike behavior with negative thermopower while for $v_{g}>0.4$ a.u. the system exhibits electronlike behavior with positive thermopower. As the gate voltage increases to $v_{g} \approx 1.4$ a.u., two electrons have been transferred

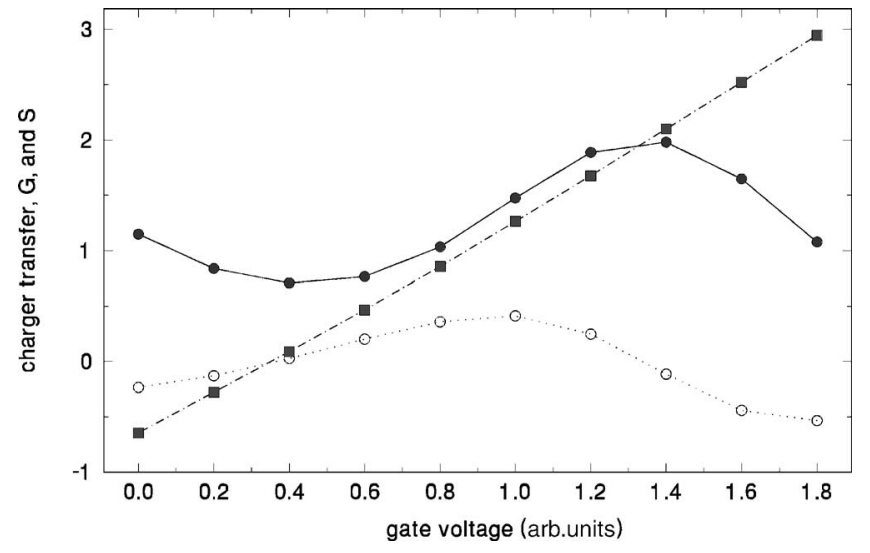

FIG. 5. The charge transfer (solid square), the electric conductance (solid circle), and the thermopower at $5 \mathrm{~K}$ (open circle) vs the gate voltage.

from the leads to the carbon atoms so that a $\pi$ state is half filled so that the conductance is maximum and the thermopower is zero again. Whenever the gate voltage sweeps across those extremum of the conductance the thermopower reverses sign.

Classically, the temperature gradient drives electrons to the low-temperature lead and the thermopower is positive which measured the effective voltage difference. Quantum mechanically, the temperature gradient may drive electrons to the high-temperature lead depending on the charge transfer. This leads to the negative thermopower which is similar to the negative effective mass of holes in the semiconductors. Negative thermopower has been found in many systems such as carbon nanotubes ${ }^{11}$ and atomic-size gold contacts using mechanically controlled break junctions. ${ }^{23}$ Recently, the even-odd parity oscillation of electric conductance has been confirmed experimentally for $\mathrm{Au}, \mathrm{Pt}$, and Ir using mechanically controlled break junctions. ${ }^{24}$ In view of the fact that the thermopower of atomic-size gold contacts has been measured using the same method as that of electric conductance, we are confident that the sign oscillation for even-odd number chains can be also tested experimentally.

In summary, we have investigated the thermoelectric transport through carbon chains connected by two $\mathrm{Al}$ electrodes. During the formation of the $\mathrm{Al}-\mathrm{C}_{n}-\mathrm{Al}$ system, charge transfer occurs and approximately an amount of $-0.5 e$ charges have been transferred from the $\mathrm{Al}$ leads to the carbon atoms. Since the transport through carbon chains is dominated by the $\pi$ states which are twofold degenerate, the thermopower exhibits a sign oscillation for even-odd number carbon chains. Since the charge transfer can be critically controlled by the gate voltage, one can tune the amount of charge transfer to be multiples of $2 e$ by varying the gate voltage. As a result, the electric conductance shows extremum and the thermopower is zero at these gate voltages leading to an oscillation as a function of gate voltage as well.

We gratefully acknowledge support by a RGC grant from the SAR Government of Hong Kong under Grant No. HKU 7044/04P and a grant from NSFC, Grant No. 10274052 (Y.D. Wei). We also thank the computer center of the University of Hong Kong for computational facilities. 
*Electronic mail: jianwang@hkusub.hku.hk

${ }^{1}$ For recent reviews, see, for example, M. A. Ratner, Mater. Today 5, (2), 20 (2002); C. Joachim, J. K. Gimzewski, and A. Aviram, Nature (London) 408, 541 (2000); J. R. Heath and M. A. Ratner, Phys. Today 56, (5), 43 (2003); A. Nitzan and M. A. Ratner, Science 300, 1384 (2003).

${ }^{2}$ N. D. Lang, Phys. Rev. B 52, 5335 (1995).

${ }^{3}$ N. D. Lang and Ph. Avouris, Phys. Rev. Lett. 81, 3515 (1998).

${ }^{4}$ N. D. Lang and Ph. Avouris, Phys. Rev. Lett. 84, 358 (2000).

${ }^{5}$ The NEGF-DFT package MCDCAL is discussed in J. Taylor, H. Guo, and J. Wang, Phys. Rev. B 63, 245407 (2001); 63, 121104(R) (2001).

${ }^{6}$ P. S. Darnle, A. W. Ghosh, and S. Datta, Phys. Rev. B 64, 201403(R) (2001).

${ }^{7}$ M. Brandbyge, J.-L. Mozos, P. Ordejón, J. Taylor, and K. Stokbro, Phys. Rev. B 65, 165401 (2002).

${ }^{8}$ Y. Xue, S. Datta, and M. A. Ratner, Chem. Phys. 281, 151 (2002)

${ }^{9}$ E. Louis, J. A. Verges, J. J. Palacios, A. J. Perez-Jimnez, and E. SanFabin, Phys. Rev. B 67, 155321 (2003).

${ }^{10}$ Y. Wei, Y. Xu, J. Wang, and H. Guo, Phys. Rev. B 70, 193406 (2004).

${ }^{11}$ K. Bradley, S. H. Jhi, P. G. Collins, J. Hone, M. L. Cohen, S. G. Louie, and A. Zettl, Phys. Rev. Lett. 85, 4361 (2000).

${ }^{12}$ D. R. Hamann, M. Schlüter, and C. Chiang, Phys. Rev. Lett. 43, 1494 (1982).

${ }^{13}$ U. Sivan and Y. Imry, Phys. Rev. B 33, 551 (1986).
${ }^{14}$ T. Rejec, A. Ramsak, and J. H. Jefferson, Phys. Rev. B 65, 235301 (2002).

${ }^{15}$ In our calculations, an Al electrode is composed of unit cells with nine $\mathrm{Al}$ atoms oriented in the (100) direction repeated to $\pm \infty$. The scattering region contains four layers of the $\mathrm{Al}$ atoms on either side of the molecule. The carbon atoms in contact with the electrodes are positioned at the hollow site for $\mathrm{Al}$ electrodes. We fixed the contact distance to be $d=1.9 \AA$ (Ref. 16). The separation between carbon atoms is $1.3225 \AA$ (Ref. 17).

${ }^{16}$ B. Larade, J. Taylor, H. Mehrez, and H. Guo, Phys. Rev. B 64, 075420 (2001).

${ }^{17}$ H. J. Choi, J. Ihm, S. G. Louie, and M. L. Cohen, Phys. Rev. Lett. 84, 2917 (2000).

${ }^{18}$ The conductance oscillation has also been found for other systems such as Na chains (Ref. 19) and Al chains (Ref. 20).

${ }^{19}$ N. D. Lang, Phys. Rev. Lett. 79, 1357 (1997); H. S. Sim et al., ibid. 87, 096803 (2001).

${ }^{20}$ K. S. Thygesen and K. W. Jacobsen, Phys. Rev. Lett. 91, 146801 (2003).

${ }^{21}$ Since the thermopower scales linearly with temperature at low temperatures, we have calculated the thermopower at $5 \mathrm{~K}$.

${ }^{22}$ Note that the carbon chains with different length have different $v_{0}$.

${ }^{23}$ B. Ludoph and J. M. van Ruitenbeek, Phys. Rev. B 59, 12290 (1999).

${ }^{24}$ R. H. M. Smit et al., Phys. Rev. Lett. 91, 076805 (2003). 